\title{
Further considerations on steady recirculating flows involving non-Newtonian fluids
}

\author{
G. Chaidron ${ }^{\mathrm{a}, *}$, F. Chinesta ${ }^{\mathrm{b}}$ \\ ${ }^{a}$ Laboratoire de Rhéologie et Thermodynamique des Matériaux Macromoléculaires, Conservatoire National des Arts et \\ Métiers, 292 rue Saint Martin, F-75141 Paris Cedex 03, France \\ ${ }^{\mathrm{b}}$ Laboratoire de Mécanique des Systémes et des Procédés, Ecole Nationale Supérieure d'Arts et Métiers, 151 Boulevard de \\ l'Hôpital, F-75013 Paris, France
}

\begin{abstract}
Numerical modeling of non-Newtonian flows typically involves the coupling between equations of motion characterized by an elliptic behavior, and the fluid constitutive equation, which is an advection equation linked to the fluid history in differential models. In a former paper, the authors have proved that linear advection equations have only one solution in steady recirculating flows. This solution is found imposing the solution periodicity along the closed streamlines of the flow. The non-linear character of constitutive equations was taken into account in a second paper. In this case, standard strategies, as Picard or Newton techniques for example, were generalized to impose the solution periodicity for each linearized problem found in the iterative procedures. In the present paper, we treat other questions related to steady recirculating flows of non-Newtonian fluids. We will prove that in some cases the steady solution of the extra-stress tensor cannot be found as the steady state associated with the evolution problem. We will also prove that only one steady solution exists for multi-mode linear differential models. Finally, we will present some results concerning integral viscoelastic models in general steady recirculating flows.
\end{abstract}

Keywords: Advection equation; Steady recirculating flows; Non-Newtonian fluid mechanics; Multi-mode constitutive models; Integral viscoelastic models

\section{Introduction}

Numerical modeling of non-Newtonian fluid flows involves usually the coupling between motion equations, which lead to an elliptic problem, and the fluid constitutive equation, which introduces an advection problem related to the fluid history.

* Corresponding author. Tel.: +33-1-40272401; fax: +33-1-40272526.

E-mail address: chaidron@cnam.fr (G. Chaidron). 


\subsection{First example: flow of short fibers suspensions}

In short fibers suspensions flow models, the extra-stress tensor $\underline{\underline{\tau}}$ depends on the fibers orientation [1-7]:

$$
\underline{\underline{\tau}}=2 \mu\left\{\underline{\underline{D}}+N_{\mathrm{p}} \operatorname{Tr}(\underline{\underline{s}} \underline{\underline{D}}) \underline{\underline{s}}\right\},
$$

where $\mu$ is the equivalent suspension viscosity, $N_{\mathrm{p}}$ the scalar parameter depending on both the fibers concentration and the fibers aspect ratio, $\underline{\underline{D}}$ the strain rate tensor, $\operatorname{Tr}($ ) the tensorial operator trace and $\underline{\underline{s}}$ is the second order orientation tensor defined by:

$$
\underline{\underline{s}}=\frac{\underline{\underline{a}}}{\operatorname{Tr}(\underline{\underline{a}})} \text {. }
$$

The evolution of the tensor $\underline{\underline{a}}$ is given by the advection equation:

$$
\frac{\mathrm{d} \underline{\underline{a}}}{\mathrm{~d} t}=\underline{\underline{\Omega}} \underline{\underline{a}}-\underline{\underline{a}} \underline{\underline{\Omega}}+k(\underline{\underline{D}} \underline{\underline{a}}+\underline{\underline{a}} \underline{\underline{D}})+D_{\mathrm{r}}\left(\underline{\underline{a}}-\frac{\underline{\underline{I}}}{3}\right),
$$

where $\Omega$ represents the vorticity tensor, $k$ a constant which depends on the fibers aspect ratio and $D_{\mathrm{r}}$ is a diffusion coefficient.

In the previous constitutive equation a quadratic closure relation has been introduced [8]. From a physical point of view, we can consider that the eigenvalues of the orientation tensor $\underline{\underline{s}}$ represent the probability of the fibers to be aligned in the direction of the corresponding eigenvectors.

The stress tensor is given by:

$$
\underline{\underline{\sigma}}=-p \underline{\underline{I}}+\underline{\underline{\tau}},
$$

where $p$ is the pressure field and $\underline{\underline{I}}$ the unit tensor.

\subsection{Second example: the upper convected Maxwell's model}

In most of the differential viscoelastic models [9-12], the evolution of the extra-stress tensor $\underline{\tau}$ is given by an advection equation. For example the upper convected Maxwell's (UCM) model is given by:

$$
\lambda \frac{\delta \underline{\underline{\tau}}}{\delta t}+\underline{\underline{\tau}}=2 \mu \underline{\underline{D}}
$$

where $\delta / \delta t$ denotes the upper convected derivative

$$
\frac{\delta \underline{\underline{\tau}}}{\delta t}=\frac{\mathrm{d} \underline{\underline{\tau}}}{\mathrm{d} t}-\operatorname{grad} \underline{v} \underline{\underline{\tau}}-\underline{\underline{\tau}}(\operatorname{grad} \underline{v})^{\mathrm{T}},
$$

and $\lambda$ is a relaxation time. The stress tensor $\underline{\underline{\sigma}}$ results in

$$
\underline{\underline{\sigma}}=-p \underline{\underline{I}}+\underline{\underline{\tau}} \text {. }
$$

The Oldroyd-B model introduces a solvent contribution

$$
\underline{\underline{\sigma}}=-p \underline{\underline{I}}+2 \mu_{\mathrm{s}} \underline{\underline{D}}+\underline{\underline{\tau}},
$$

where $2 \mu_{\mathrm{s}} \underline{\underline{D}}$ represents the Newtonian contribution of the solvent in the polymer solution. 
Many of differential models currently used in numerical simulations can be written in the general form [9]:

$$
\lambda \frac{\delta \underline{\underline{\underline{\tau}}}}{\delta t}+A(\underline{\underline{\tau}}) \underline{\underline{\tau}}=2 \mu \underline{\underline{D}}
$$

\subsection{Third example: the viscoelastic Pom-Pom model}

In the recent Pom-Pom viscoelastic model, as described in [10], the extra-stress tensor depends on both the molecular orientation $\underline{\underline{s}}$ and the molecular extension $\lambda$ :

$$
\underline{\underline{\tau}}=\alpha \lambda^{2} \underline{\underline{s}}
$$

where $\alpha$ is a material parameter.

The evolutions of the two variables are also governed by the following advection equations:

$$
\begin{aligned}
& \frac{\mathrm{d} \underline{\underline{a}}}{\mathrm{~d} t}=\underset{\underline{\Omega}}{\underline{\underline{a}}} \underline{\underline{a}}-\underline{\underline{a}} \underline{\underline{\Omega}}+\underline{\underline{D}} \underline{\underline{a}}+\underline{\underline{a}} \underline{\underline{D}}-\frac{1}{\tau_{\mathrm{b}}}\left(\underline{\underline{a}}-\frac{\underline{\underline{I}}}{3}\right), \\
& \underline{\underline{s}}=\frac{\underline{\underline{a}}}{\operatorname{Tr}(\underline{\underline{s}})},
\end{aligned}
$$

and

$$
\frac{\mathrm{d} \lambda}{\mathrm{d} t}=\lambda \operatorname{Tr}(\underline{\underline{s}} \underset{\underline{D}}{)})-\frac{1}{\tau_{\mathrm{s}}}(\lambda-1),
$$

where $\tau_{\mathrm{b}}$ and $\tau_{\mathrm{s}}$ are two relaxation times.

The stress tensor is given by:

$$
\underline{\underline{\sigma}}=-p \underline{\underline{I}}+\underline{\underline{\tau}}
$$

\subsection{The problematic related to steady recirculating flows}

For the models described an uncoupled resolution can be carried out. Thus, we can identify an elliptic problem related to the flow kinematics:

$$
\left\{\begin{array}{l}
\operatorname{div} \underline{\underline{\sigma}}=\underline{0}, \\
\operatorname{div} \underline{v}=0, \\
\underline{\underline{\sigma}}=-p \underline{\underline{I}}+2 \mu_{\mathrm{s}} \underline{\underline{D}}+\underline{\underline{\tau}},
\end{array}\right.
$$

where $\underline{\underline{\tau}}$ can be taken from the previous iteration. When the model does not involve a Newtonian contribution, i.e. when the stress tensor is given by $\underline{\sigma}=-p \underline{I}+\underline{\tau}$, the ellipticity is retained in the formulation by using an elastic viscous stress splitting (EVSS) [11] or its discrete variant, the discrete elastic viscous stress splitting (DEVSS), which introduces the strain tensor as a new model variable [12] (a particular DEVSS formulation for the Pom-Pom model is given in [13]). In this case, the flow kinematics are 
defined by:

$$
\left\{\begin{array}{l}
\operatorname{div} \underline{\underline{\sigma}}=\underline{0}, \\
\operatorname{div} \underline{v}=0, \\
\underline{\underline{\sigma}}=-p \underline{\underline{I}}+2 \mu_{\mathrm{a}} \underline{\underline{D}}-2 \mu_{\mathrm{a}} \underline{\underline{D}} \overline{+} \underline{\underline{\tau}}, \\
\underline{\tilde{D}}=\frac{1}{2}\left(\operatorname{grad} \underline{v}+(\operatorname{grad} \underline{v})^{\mathrm{T}}\right) .
\end{array}\right.
$$

A standard finite element strategy may be applied to solve this equation. The only difficulty is to choose cleverly the functional spaces required to approximate the velocity, pressure, stress and strain fields [12].

Actually, difficulty appears when we integrate the advection equation governing the evolution of the extra-stress tensor, which defines an hyperbolic problem. When we consider the integration of this type of equations in a steady recirculating flow, the problem seems a priori ill defined, because neither initial nor boundary conditions are given. However, in a former paper, the authors have proved that linear advection equations in general steady recirculating flows have only one solution that can be found by imposing the periodicity along the closed streamlines of the flow [14].

Sometimes, the steady solution can be found by the integration of the evolution equation, using the method of characteristics, until reaching the steady state. However, as we prove in the present paper, the steady solution can only be reached for a particular choice of the initial conditions. For other initial conditions, the steady state does not exist.

The non-linear character of constitutive equations was considered in [15]. In this case, standard strategies, as Picard or Newton techniques for example, were generalized to impose the solution periodicity for each one of the linearized problems found in the iterative procedures. However, for non-linear models, we cannot conclude about the unicity of the periodic solution found by any of the previous strategies.

In this paper, we emphasize on other questions related to steady recirculating flows of non-Newtonian fluids. We show that in some cases the steady state of the extra-stress cannot be found as the steady solution of the associated evolution problem. We also prove that only one steady solution of the extra-stress tensor exists for linear multi-mode differential models, this solution being characterized by the periodicity of each partial extra-stress. Finally, we will present some results about the integral UCM viscoelastic model in steady recirculating flows.

As a consequence of one of our previous papers [14], number of colleagues ask for the possibility of application in different cases of our strategy to solve transport equation in steady recirculating flows. We then try, in this work, to deal with three specific problems related to the application of this numerical strategy. For this reason, the three main parts of this paper can be seen as independent from each other.

\subsubsection{Notation}

- We will denote a vector by an underline, e.g. $\underline{v}$, with components $v_{i}$.

- In the same way, the components of a second order tensor $\underline{\underline{a}}$ will be expressed as $a_{i j}$.

\section{Steady solution of linear advection equations in steady recirculating flows}

In general, the advection equations treated in this paper may be written in the form:

$$
\frac{\mathrm{d} \underline{a}}{\mathrm{~d} t}=\underline{A}(\underline{x}(t)) \underline{a}+\underline{B}(\underline{x}(t)),
$$


where

$$
\underline{a}=\left(\begin{array}{c}
a_{1} \\
a_{2} \\
\vdots \\
a_{n}
\end{array}\right)
$$

represents the column vector form of a generic tensor. The matrix $\underset{=}{A}$ and the vector $\underline{B}$ depend on the material properties as well as on the flow kinematics. If we assume a steady regime, the flow kinematics is a function of the particle position given by the integration of:

$$
\frac{\mathrm{d} x}{\mathrm{~d} t}=\underline{v}(\underline{x}(t)),
$$

which represents the characteristics of Eq. (17).

In [14], the authors gave a numerical procedure in order to determine the solution verifying the periodicity condition, which is required if one is looking for a steady solution. We summarize here the main ideas.

The differential system given by Eq. (17) is linear and in consequence its general solution may be written as the addition of the general solution of the homogeneous differential equation:

$$
\frac{\mathrm{d} \underline{a}}{\mathrm{~d} t}=\underline{A}(\underline{x}(t)) \underline{a}
$$

and a particular solution of the non-homogeneous differential equation (Eq. (17)).

As a linear combination of solutions is also a solution of the homogeneous equation, we can integrate along a closed streamline by the method of characteristics, the following $n$ advection equations:

$$
\frac{\mathrm{d} \underline{a}_{\mathrm{h}}^{k}}{\mathrm{~d} t}=\underline{A}(\underline{x}(t)) \underline{a}_{\mathrm{h}}^{k}, \quad \forall k \in[1, \ldots, n],
$$

with the initial conditions given by

$$
\underline{a}_{\mathrm{h}}^{k}(t=0)=\left(\begin{array}{c}
a_{1} \\
a_{2} \\
\vdots \\
a_{k-1} \\
a_{k} \\
a_{k+1} \\
\vdots \\
a_{n}
\end{array}\right)=\left(\begin{array}{c}
0 \\
0 \\
\vdots \\
0 \\
1 \\
0 \\
\vdots \\
0
\end{array}\right)
$$

The solutions of these equations are noted by $\underline{a}_{\mathrm{h}}^{k}$, where the indice $\mathrm{h}$ indicates that these solutions are related to the homogeneous equation. Now, we can express the general solution $\underline{a}_{\mathrm{h}}$ of the homogeneous 
equation, as indicated, from a linear combination, i.e.

$$
\underline{a}_{\mathrm{h}}=\sum_{k=1}^{k=n} \alpha_{k} \underline{a}_{\mathrm{h}}^{k}
$$

where $\alpha_{k}, k=[1, \ldots, n]$, represent the $n$ unknown scalar parameters.

To obtain a particular solution $\underline{a}_{\mathrm{p}}$ of Eq. (17), we integrate this equation from an arbitrary initial condition, for example $\underline{a}_{\mathrm{p}}(t=0)=\underline{0}$. The general solution of the linear advection equation $\underline{a}$ is then:

$$
\underline{a}=\underline{a}_{\mathrm{h}}+\underline{a}_{\mathrm{p}}=\underline{a}_{\mathrm{p}}+\sum_{k=1}^{k=n} \alpha_{k} \underline{a}_{\mathrm{h}}^{k}
$$

Now if we are looking for a periodic solution, we have to impose

$$
\underline{a}(t=0)=\underline{a}(t=T),
$$

where $T$ is the time period, i.e. the time that an imaginary particle takes to come back to its starting point after a turn.

This periodicity condition, which is a necessary condition for a steady state regime, implies:

$$
\underline{\alpha}=\underline{a}_{\mathrm{p}}(t=0)+\sum_{k=1}^{k=n} \alpha_{k} \underline{a}_{\mathrm{h}}^{k}(t=0)=\underline{B} \underline{\alpha}+\underline{a}_{\mathrm{p}}(t=T),
$$

where the $k$ th column of $\underline{B}$ represents the solution of $\underline{a}_{\mathrm{h}}^{k}$ at time $t=T$, and the vector $\underline{\alpha}$ is defined by:

$$
\underline{\alpha}=\left(\begin{array}{c}
\alpha_{1} \\
\alpha_{2} \\
\vdots \\
\alpha_{n}
\end{array}\right)
$$

Thus, the linear algebraic system, Eq. (26), can be written as:

$$
(\underline{B}-\underline{\underline{I}}) \underline{\alpha}=-\underline{a}_{\mathrm{p}}(t=T) .
$$

For general flows, $\operatorname{det}(\underline{B}-\underline{\underline{I}}) \neq 0$ and $\underline{a}_{\mathrm{p}}(t=T) \neq \underline{0}$ [14]. Thus, the previous system has only one solution $\underline{\alpha}$, which defines the solution to be considered as the initial condition in order to obtain a periodic solution:

$$
\underline{a}(t=0)=\underline{\alpha} .
$$

If we integrate now Eq. (17) by the method of characteristics along a closed streamline, from the previous initial condition $(\underline{a}(t=0)=\underline{\alpha})$, we obtain the only solution which verifies $\underline{a}(t=0)=\underline{a}(t=T)$ and which is the steady solution of our linear advection problem.

Remark 1. If we impose the periodicity condition $\underline{a}(t=0)=\underline{a}(t=T)$, we obtain a solution which reaches the value $\underline{a}(t=0)$ after each turn, i.e. for $t=n T$ with $n \in \mathbb{N}$. However, if we impose a periodicity 
in the form $\underline{a}(t=0)=\underline{a}(t=n T)$ with $n>1$, we obtain a solution $n T$-periodic but which is not in general the steady solution. To prove this result, we proceed as previously imposing in this case:

$$
\underline{a}(t=0)=\underline{a}(t=n T), \quad n>1,
$$

from which we obtain the linear system

$$
(\underline{\tilde{B}}-I) \underline{\tilde{\alpha}}=-\underline{a}_{\mathrm{p}}(t=n T),
$$

where the $k$ th column of $\underline{\underline{B}}$ is the solution of a $\underline{a}_{\mathrm{h}}^{k}$ at time $t=n T$.

Generally, $\underline{\tilde{\alpha}}$ becomes different from $\underline{\alpha}$. As indicated previously, $\underline{\alpha}$ is the unique initial condition for which $\underline{a}(t=0)=\underline{a}(t=T)$. Thus, if we consider $\underline{\tilde{\alpha}}$ as initial condition, we obtain after a turn $\underline{a}(t=0) \neq \underline{a}(t=T)$, which proves that $\underline{\tilde{\alpha}}$ does not define the steady solution.

\section{Steady solutions versus steady state of evolution problems}

From the previous explanation, one could think that the search for periodicity to obtain a steady solution of a linear advection equation is equivalent to solve the advection problem (Eq. (17)) by the method of characteristics along a closed streamline until reaching the steady state regime, expected after some turns [16].

In this section we prove the existence of situations in contradiction with the precedent assumption. Let us consider the flow of a short fibers suspension without any diffusion effect and where the fibers have a finite aspect ratio.

The extra-stress tensor evolution is given by Eq. (3) with $D_{\mathrm{r}}=0$ :

$$
\frac{\mathrm{d} \underline{\underline{a}}}{\mathrm{~d} t}=\underline{\underline{\Omega}} \underline{\underline{a}}-\underline{\underline{a}} \underline{\underline{\Omega}}+k(\underline{\underline{D}} \underline{\underline{a}}+\underline{\underline{a}} \underline{\underline{D}}),
$$

with the orientation tensor $\underline{\underline{s}}$ given by

$$
\underline{\underline{s}}=\frac{\underline{a}}{\operatorname{Tr}(\underline{a})} .
$$

We have proved in [17] that for a general flow of a suspension of fibers of finite aspect ratio there is no solution verifying

$$
\max _{j}\left(\lambda_{j}\right)=1,
$$

where $\lambda_{j}$ are the eigenvalues of the tensor $\underline{s}$. Actually, the meaning of this result is that the probability to find a fiber, in a point of the flow, aligned in the direction of the eigenvector associated with the higher eigenvalue is strictly lower than 1. Thus, a light misalignment remains. This result was verified in [14], using the numerical procedure previously described to obtain the steady solution.

For example if we consider the planar flow given by the following velocity field:

$$
\underline{v}^{\mathrm{T}}=\left(-y \sqrt{x^{2}+y^{2}}, x \sqrt{x^{2}+y^{2}}\right),
$$

the velocity is constant on a circular trajectory centered in the point $(0,0)$ and its magnitude is the radius squared. We are looking for a periodic orientation solution with a unit trace at the point $\underline{x}^{\mathrm{T}}=(0,1)$ by 


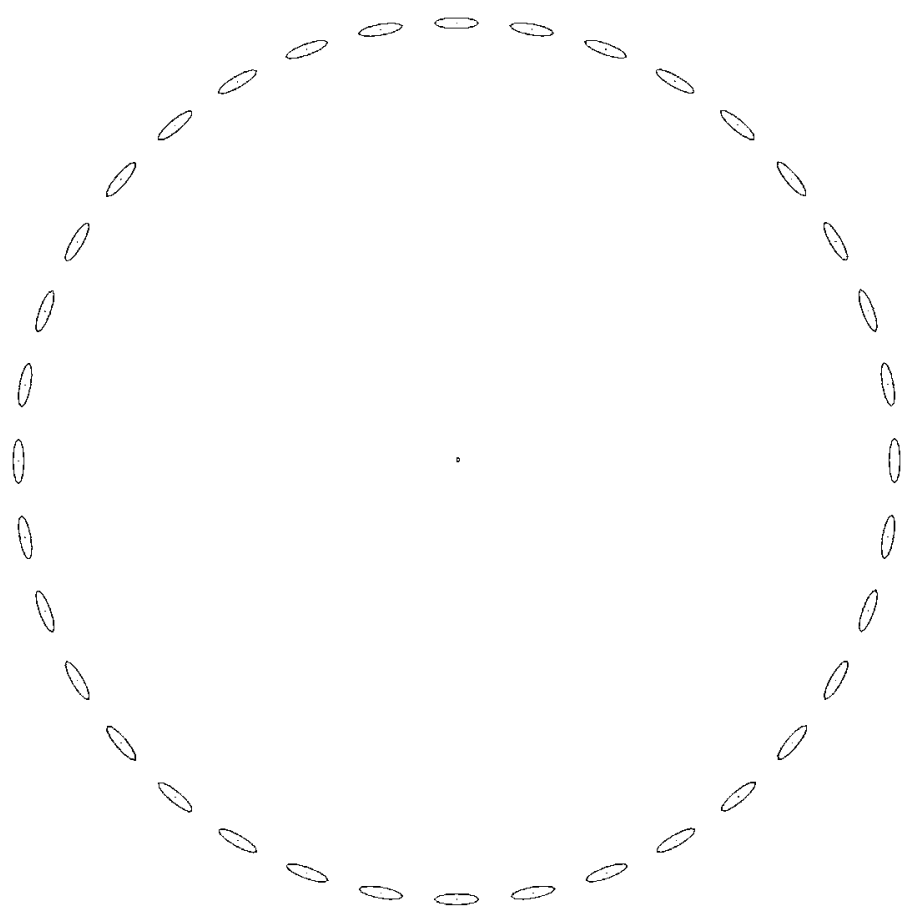

Fig. 1. Steady orientation for fibers with a finite aspect ratio.

using the strategy described previously, for fibers with $k=0.6$. The solution found at the point $\underline{x}^{\mathrm{T}}=(0,1)$ is given by:

$$
\underline{\underline{S}}_{(0,1)}=\left(\begin{array}{cc}
0.8 & 0 \\
0 & 0.2
\end{array}\right) .
$$

The periodic solution associated with the initial condition given by Eq. (36) along a streamline is depicted in Fig. 1, where we represent the orientation tensor by an ellipse. The ellipse axes directions correspond to the eigenvectors of the orientation tensor and the half of the axes length correspond to the eigenvalues. We can see, in this figure, that the ellipses have a certain thickness corresponding of a non-null eigenvalue in the radial direction. That means that the most probable direction of a fiber is the direction of the streamline but a light misalignment exists.

To find the steady solution of Eq. (32) from an evolution procedure we need to integrate it from an arbitrary initial condition until reaching the steady state. This steady regime is reached when the solution does not vary with time in any point. However, when the orientation tensor considered as the initial condition has an eigenvalue equal to 1 , i.e. $\max _{j}\left(\lambda_{j}\right)=1$, then the solution at time $t, \underline{\underline{s}}(t)$, has also an eigenvalue equal to 1 .

To prove this result we consider an equivalent form of Eqs. (32) and (33):

$$
\frac{\mathrm{d} \underline{\underline{s}}}{\mathrm{~d} t}=\underline{\underline{\Omega}} \underline{\underline{s}}-\underline{\underline{\underline{S}}} \underline{\underline{\Omega}}+k(\underline{\underline{D}} \underline{\underline{s}}+\underline{\underline{\underline{S}}} \underline{\underline{D}}-2 \operatorname{Tr}(\underline{\underline{s}} \underline{\underline{D}}) \underline{\underline{s}}) .
$$


It is easy to verify that the solution of Eq. (37) may be written in the form:

$$
\underline{\underline{s}}=\frac{\underline{F}_{k} \underline{S}_{0} \underline{F}_{k}^{\mathrm{T}}}{\operatorname{Tr}\left(\underline{\underline{F}}_{k} \underline{S}_{0} \underline{F}_{k}^{\mathrm{T}}\right)},
$$

where $\underline{\underline{S}}_{0}$ is the initial orientation tensor and the transport $\underline{F}_{k}$ verifies the following linear advection equation:

$$
\frac{\mathrm{d} \underline{\underline{F}}}{\mathrm{~d} t}=\operatorname{grad} \underline{v} \underline{F}_{k},
$$

with the initial condition given by:

$$
\underline{F}_{k}(t=0)=\underline{\underline{I}} .
$$

Now, we consider an initial orientation tensor $\underline{\underline{s}}_{0}$ whose maximum eigenvalue is equal to 1 . If we express $\underline{S}_{0}$ in the system of coordinates associated with the eigenvectors of $\underline{S}_{0}$, the expression of $\underline{\underline{S}}_{0}$ results in:

$$
\underline{s}_{0}=\left(\begin{array}{ll}
1 & 0 \\
0 & 0
\end{array}\right),
$$

that, introduced in Eq. (38), gives the expression of the tensor $\underline{\underline{s}}$ at time $t$

$$
\underline{\underline{s}}=\frac{1}{F_{11}^{2}+F_{21}^{2}}\left(\begin{array}{ll}
F_{11}^{2} & F_{11} F_{21} \\
F_{11} F_{21} & F_{21}^{2}
\end{array}\right),
$$

where $F_{i j}$ denotes the components of tensor $\underline{F}_{k}$. If we determine the eigenvalues of $\underline{\underline{s}}$ from the previous expression, Eq. (42), we obtain $\lambda_{1}=1$ and $\lambda_{2}=0$, which confirm our assumption.

In this case, it is impossible to reach the steady solution associated with the initial orientation given by Eq. (36) (which is shown in Fig. 1). As we have proved that the solution associated to Eq. (36) is the only steady solution, we can conclude that the solution of Eq. (37) will not reach a steady state solution for every initial conditions.

As it is well established the solution of a hyperbolic equation, even if there is only one, cannot be obtained from any initial condition.

Remark 2. As the orientation tensor given by Eq. (36) is the solution of Eq. (37) or its equivalent formulation given by Eqs. (32) and (33), this orientation state depends on the quadratic closure relation considered. From a physical point of view, the quadratic closure relation gives an exact solution of the orientation tensor when the fibers are locally perfectly aligned, i.e. when the eigenvalues of this tensor are 1 and 0 in a 2D case. The solution of the orientation tensor given by Eq. (36) is not that so. Thus, this solution can be not exact from a physical point of view. However, we have shown that fibers remain locally aligned if they were locally aligned at the initial state, and in this case the quadratic closure relation is exact. Thus, that means that the steady solution cannot be obtained from an arbitrary initial condition (in particular from an initial locally aligned state). 


\section{Linear multi-mode constitutive equations}

The generic differential constitutive equation for a viscoelastic fluid, Eq. (9), can be extended to the case of a spectrum of relaxation times writing:

$$
\underline{\underline{\tau}}=\sum_{j=1}^{j=m} \underset{\underline{\tau}}{=}
$$

where each partial extra-stress tensor $\underset{\underline{\underline{\tau}}}{j}$ obeys to an advection equation:

$$
\lambda_{j} \frac{\delta \underline{\underline{\underline{\tau}}} j}{\delta t}+A \underline{\underline{\tau}} j=2 \mu_{j} \underline{\underline{D}},
$$

with material coefficients $\lambda_{j}$ and $\mu_{j}$.

In this section we prove that only one steady solution exists for the problem defined by Eqs. (43) and (44) in general steady recirculating flows. We can construct a solution $\underline{\underline{\tau}}$ verifying the periodicity restriction in the following way:

$$
\underline{\underline{\tau}}(t=0)=\underline{\underline{\tau}}(t=T),
$$

which can be rewritten in the form:

$$
\sum_{j=1}^{j=m} \underline{\underline{\tau}}_{j}(t=0)=\sum_{j=1}^{j=m} \underline{\underline{\tau}}_{j}(t=T) .
$$

The $\underline{\underline{\tau}}_{j}^{0}$ is the initial condition for the first $m-1$ modes, i.e. $\underline{\underline{\tau}}_{j}(t=0)=\underline{\underline{\tau}}_{j}^{0}, \forall j \in[1, \ldots, m-1]$. The solution obtained by integrating Eq. (44) from the initial condition $\underline{\tau}_{j}^{0}$ is denoted by $\underline{\tau}_{j}$ whose value after a complete rotation becomes $\underline{\underline{\tau}}_{j}(t=T)=\underline{\underline{\tau}}_{j}^{\mathrm{T}}$. We consider the case where these solutions and their sum are not periodic. Now, the initial value for the last mode $\underline{\underline{\tau}}_{m}$ is chosen in order to verify Eq. (45).

From the procedure previously described, and writing every tensor as a column vector, we have:

$$
\sum_{j=1}^{j=m-1} \underline{\tau}_{j}^{0}+\underline{\alpha}=\sum_{j=1}^{j=m-1} \underline{\tau}_{j}^{\mathrm{T}}+\underline{\underline{B}} \underline{\alpha}+\underline{\tau}_{\mathrm{p}}(t=T),
$$

where the $k$ th column of $\underline{B}$ represents the solution at time $t=T$ of the homogeneous equation related to the $m$-mode:

$$
\lambda_{m} \frac{\delta \underline{\underline{\tau}}_{m}}{\delta t}+A \underline{\underline{\tau}}_{m}=0
$$


with the initial condition given by:

$$
\underline{\tau}_{m}(t=0)=\left(\begin{array}{c}
\tau_{1} \\
\tau_{2} \\
\vdots \\
\tau_{k-1} \\
\tau_{k} \\
\tau_{k+1} \\
\vdots \\
\tau_{n}
\end{array}\right)=\left(\begin{array}{c}
0 \\
0 \\
\vdots \\
0 \\
1 \\
0 \\
\vdots \\
0
\end{array}\right),
$$

and $\underline{\tau}_{\mathrm{p}}(t=T)$ represents the solution of the general equation:

$$
\lambda_{m} \frac{\delta \underline{\underline{\tau}}}{\delta t}+A \underline{\underline{\tau}} m=2 \mu_{m} \underline{\underline{D}}
$$

when a null vector is imposed as initial condition $\left(\underline{\tau}_{\mathrm{p}}(t=0)=\underline{0}\right)$.

From Eq. (47) we can compute the components of the vector $\underline{\alpha}$ ( $\underline{\alpha}$ in a tensor form) which is the initial condition to be imposed to the $m$-mode in order to insure

$$
\underline{\underline{\tau}}(t=0)=\underline{\underline{\tau}}(t=T) .
$$

Now, we are going to prove that even if the set of initial conditions:

$$
\left\{\underline{\underline{\tau}}_{1}(t=0), \ldots, \underline{\underline{\tau}}_{m-1}(t=0), \underline{\underline{\alpha}}\right\},
$$

allows us to obtain a solution of $\underline{\underline{\tau}}$ verifying Eq. (52), this solution is not necessary periodic. Thus, in general:

$$
\underline{\underline{\tau}}(t=0)=\underline{\underline{\tau}}(t=T) \neq \underline{\underline{\tau}}(t=2 T) .
$$

This can be demonstrated as presented in the following lines. Because we do not impose the periodicity of the first $m-1$ modes:

$$
\sum_{j=1}^{j=m-1} \underline{\tau}_{j}^{0}-\sum_{j=1}^{j=m-1} \underline{\tau}_{j}^{\mathrm{T}} \neq \underline{0} .
$$

Furthermore, in the second turn, $t \in[T, 2 T]$, we have

$$
\sum_{j=1}^{j=m-1} \underline{\tau}_{j}^{\mathrm{T}}+\underline{\alpha}^{*}=\sum_{j=1}^{j=m-1} \underline{\tau}_{j}^{2 \mathrm{~T}}+\underline{B} \underline{\alpha}^{*}+\underline{\tau}_{\mathrm{p}}(t=T),
$$

where $\underline{\tau}_{j}^{2 \mathrm{~T}}$ represents the value after a second turn for the $j$-mode solution, associated to the initial value $\underline{\tau}_{j}^{\mathrm{T}}$ at the beginning of this turn $(t=T)$. On the other hand, $\underline{\alpha}^{*}$ is the initial value to be imposed to the $m$-mode $\left(\underline{\tau}_{m}(t=T)\right)$ in order to insure

$$
\underline{\underline{\tau}}(t=T)=\underline{\underline{\tau}}(t=2 T) \text {. }
$$


We can now analyze which conditions on the initial value are required for $m$-mode in the second turn to be equal to its final value at the first turn. These conditions are necessary to insure

$$
\underline{\underline{\tau}}(t=0)=\underline{\underline{\tau}}(t=T)=\underline{\underline{\tau}}(t=2 T) .
$$

From Eq. (47) we obtain the expression of $\alpha$ :

$$
\underline{\alpha}=(\underline{B}-\underline{I})^{-1}\left\{\sum_{j=1}^{j=m-1} \underline{\tau}_{j}^{0}-\sum_{j=1}^{j=m-1} \underline{\tau}_{j}^{\mathrm{T}}-\underline{\tau}_{\mathrm{p}}(t=T)\right\} .
$$

Thus, the final value after the first turn $(t=T)$ for the $m$-mode is

$$
\underline{\tau}_{m}(t=T)=\underline{\tau}_{\mathrm{p}}(t=T)+\underline{\underline{B}} \underline{\alpha}=\underline{\tau}_{\mathrm{p}}(t=T)+\underline{\underline{B}}(\underline{\underline{B}}-\underline{I})^{-1}\left\{\sum_{j=1}^{j=m-1} \underline{\tau}_{j}^{0}-\sum_{j=1}^{j=m-1} \underline{\tau}_{j}^{\mathrm{T}}-\underline{\tau}_{\mathrm{p}}(t=T)\right\} .
$$

We obtain the expression of $\underline{\alpha}^{*}$ from Eq. (55):

$$
\underline{\alpha}^{*}=(\underline{\underline{B}}-\underline{\underline{I}})^{-1}\left\{\sum_{j=1}^{j=m-1} \underline{\tau}_{j}^{\mathrm{T}}-\sum_{j=1}^{j=m-1} \underline{\tau}_{j}^{2 \mathrm{~T}}-\underline{\tau}_{\mathrm{p}}(t=T)\right\} .
$$

Thus, the initial value in the second turn $(t=T)$ for the $m$-mode is

$$
\underline{\tau}_{m}(t=T)=\underline{\alpha}^{*}=(\underline{\underline{B}}-\underline{\underline{I}})^{-1}\left\{\sum_{j=1}^{j=m-1} \underline{\tau}_{j}^{\mathrm{T}}-\sum_{j=1}^{j=m-1} \underline{\tau}_{j}^{2 \mathrm{~T}}-\underline{\tau}_{\mathrm{p}}(t=T)\right\} .
$$

If Eqs. (59) and (61) are equal, then the continuity of the $m$-mode solution is verified. We thus write:

$$
\begin{aligned}
& \underline{\underline{B}}\left(\underline{\underline{B}}-\underline{\underline{I}}^{-1}\left\{\sum_{j=1}^{j=m-1} \underline{\tau}_{j}^{0}-\sum_{j=1}^{j=m-1} \underline{\tau}_{j}^{\mathrm{T}}-\underline{\tau}_{\mathrm{p}}(t=T)\right\}\right. \\
& \quad+\underline{\tau}_{\mathrm{p}}(t=T)=(\underline{B}-\underline{\underline{I}})^{-1}\left\{\sum_{j=1}^{j=m-1} \underline{\tau}_{j}^{\mathrm{T}}-\sum_{j=1}^{j=m-1} \underline{\tau}_{j}^{2 \mathrm{~T}}-\underline{\tau}_{\mathrm{p}}(t=T)\right\},
\end{aligned}
$$

which is equivalent to

$$
\left\{\sum_{j=1}^{j=m-1} \underline{\tau}_{j}^{\mathrm{T}}-\sum_{j=1}^{j=m-1} \underline{\tau}_{j}^{2 \mathrm{~T}}\right\}=(\underline{\underline{B}}-\underline{\underline{I}}) \underline{\underline{B}}(\underline{\underline{B}}-\underline{\underline{I}})^{-1}\left\{\sum_{j=1}^{j=m-1} \underline{\tau}_{j}^{0}-\sum_{j=1}^{j=m-1} \underline{\tau}_{j}^{\mathrm{T}}\right\} .
$$

Unfortunately, this equality is generally not verified. Consequently, the only general choice consists in imposing the periodicity for each partial extra-stress tensor. In this case we have

$$
\left\{\sum_{j=1}^{j=m-1} \underline{\tau}_{j}^{\mathrm{T}}-\sum_{j=1}^{j=m-1} \underline{\tau}_{j}^{2 \mathrm{~T}}\right\}=\underline{0},
$$


and

$$
\left\{\sum_{j=1}^{j=m-1} \underline{\tau}_{j}^{0}-\sum_{j=1}^{j=m-1} \underline{\tau}_{j}^{\mathrm{T}}\right\}=\underline{0},
$$

verifying Eq. (63).

Thus, the steady solution of the multi-mode model is obtained by solving the steady linear problem related to each mode, and for each of these problems the accurate technique described in Section 2 can be applied successfully.

Remark 3. This result can be obtained differently than described previously considering the following multi-mode scalar problem:

$$
\left\{\begin{array}{l}
\phi=\sum_{j=1}^{j=m} \phi^{j}, \\
\frac{\mathrm{d} \phi^{j}}{\mathrm{~d} t}+a^{j} \phi^{j}=b^{j},
\end{array}\right.
$$

where $a^{j}$ and $b^{j}$ depend on the flow variables and the considered mode, but they do not depend on $\phi^{j}$. We also assume that the solutions $\phi^{j}$ are independent.

In this case, the periodicity condition required for $\phi$ can be written as:

$$
\sum_{j=1}^{j=m} \phi^{j}(t)=\sum_{j=1}^{j=m} \phi^{j}(t+T), \quad \forall t .
$$

We can define the functions $\varphi^{k}(t)$ as:

$$
\varphi^{k}(t)=\phi^{j}(t)-\phi^{j}(t+T),
$$

for which the periodicity of $\phi$ results in:

$$
\sum_{j=1}^{j=m} \varphi^{j}(t)=0, \quad \forall t .
$$

This implies that the $k$-mode solution can be written as:

$$
\varphi^{k}(t)=-\sum_{j=1, j \neq k}^{j=m} \varphi^{j}(t), \quad \forall t,
$$

that is forbidden by the independence of the solutions of each mode.

Remark 4. For non-linear multi-mode viscoelastic models, a steady solution of the non-linear advection equation related to each mode can be computed using the techniques described in [15]. A solution for the extra-stress tensor may be written as the sum of the steady solutions for each mode. In this case, we cannot conclude about the unicity of the solution. 


\section{The integral upper convected Maxwell model in steady recirculating flows}

As indicated by Peters et al. [18], differential constitutive equations have the numerical advantage that the stress evolution at time $t$ depends only on the current kinematics and stresses. However, many successful constitutive equations are integral. We will consider in this section an Eulerian/ Lagrangian uncoupled approach for steady recirculating flows. The flow kinematics results from the discretization of the Eulerian formulation of the equations of motion. From this kinematics, we can compute the steady stress field by the integration of constitutive equation given by an integral relation.

In order to compare the results obtained from the integral and the differential constitutive equations, we choose a model which can be easily written in both ways, the UCM model. The differential form of the UCM model, given by Eq. (5):

$$
\lambda \frac{\delta \underline{\underline{\underline{\tau}}}}{\delta t}+\underline{\underline{\tau}}=2 \mu \underline{\underline{D}}
$$

is solved using the numerical procedure described in Section 2. The integral counterpart of the UCM model is given by:

$$
\underline{\underline{\tau}}(t)=\frac{\mu}{\lambda} \underline{I}+\frac{\mu}{\lambda^{2}} \int_{-\infty}^{t} \mathrm{e}^{\left(t^{\prime}-t\right) / \lambda} \underline{\underline{B}}\left(t, t^{\prime}\right) \mathrm{d} t^{\prime},
$$

where $\underline{\underline{B}}\left(t, t^{\prime}\right)$ is the Finger's strain tensor (inverse of the Cauchy-Green strain tensor $\underline{\underline{C}}\left(t, t^{\prime}\right)$ ):

$$
\underline{\underline{B}}\left(t, t^{\prime}\right)=\underline{\underline{C}}^{-1}\left(t, t^{\prime}\right),
$$

with

$$
\underline{\underline{C}}\left(t, t^{\prime}\right)=\underline{\underline{F}}^{\mathrm{T}}\left(t, t^{\prime}\right) \underline{\underline{F}}\left(t, t^{\prime}\right),
$$

and where the deformation gradient $\underline{\underline{F}}$ obeys the following transport equation:

$$
\left\{\begin{array}{l}
\frac{\mathrm{d} \underline{\underline{F}}}{\mathrm{~d} t^{\prime}}=\operatorname{grad} \underline{v} \underline{\underline{F}} \\
\underline{\underline{F}}\left(t^{\prime}=t\right) \underline{\underline{I}}
\end{array}\right.
$$

Now, we are going to prove that, for steady recirculating flows the solution, $\underline{\underline{\tau}}(t)$ obtained by the integration of Eq. (72) verifies $\underline{\underline{\tau}}(t)=\underline{\underline{\tau}}(t+T)$, where $T$ is the rotation period. Consequently, for each point $\underline{x}$ inside the recirculating flow domain, we can consider an arbitrary time $t$ and integrate Eq. (72) by the method of characteristics to obtain $\underline{\underline{\tau}}(\underline{x})$. At the same point, one period later $(t+T)$, the solution remains unchanged. The periodicity is thus implicitly verified.

In order to prove the equality $\underline{\underline{\tau}}(t)=\underline{\underline{\tau}}(t+T)$, we give two intermediate results:

(1) The first one concerns the gradient of deformation $\underset{\underline{F}}{\underline{ }}\left(t, t^{\prime}\right)$ at time $t^{\prime}$. As the equation that governs the evolution of $\underline{\underline{F}}\left(t, t^{\prime}\right)$ is linear and homogeneous, we can write the solution at time $t^{\prime}$ in the vector form:

$$
\underline{\underline{F}}\left(t, t^{\prime}\right)=\underline{\underline{M}}\left(t, t^{\prime}\right) \underline{\underline{F}}\left(t, t^{\prime}\right)=\underline{\underline{M}}\left(t, t^{\prime}\right) \underline{I},
$$


where $\underline{I}$ is the vector form of the unit tensor, and $M\left(t, t^{\prime}\right)$ depends on the flow kinematics along the imaginary path between $\underline{x}(t)$ and $\underline{x}\left(t^{\prime}\right)$. Because the flow is steady and recirculating, we have:

$$
\underline{\underline{M}}\left(t, t^{\prime}\right)=\underline{\underline{M}}\left(t+T, t^{\prime}+T\right),
$$

and, as a direct consequence:

$$
\underline{\underline{B}}\left(t, t^{\prime}\right)=\underline{\underline{B}}\left(t+T, t^{\prime}+T\right) .
$$

(2) The second result concerns the behavior of $\underline{B}\left(t, t^{\prime}\right)$ when $t^{\prime}$ approaches $-\infty$ in steady recirculating flows. If we start from

$$
\underline{F}(t, t-T)=\underline{M}(t, t-T) \underline{I},
$$

using again the steady and recirculating flow behavior again, we have:

$$
\underline{F}(t, t-2 T)=\underline{M}(t-T, t-2 T) \underline{F}(t, t-T) .
$$

If we use now Eq. (77), we can write the previous relation as:

$$
\underline{F}(t, t-2 T)=\underline{M}(t, t-T) \underline{F}(t, t-T),
$$

which, using Eq. (79), results in:

$$
\underline{F}(t, t-2 T)=\underline{M}^{2}(t, t-T) \underline{I} .
$$

This expression can be generalized for $n$ turns:

$$
\underline{F}(t, t-n T)=\underline{M}^{n}(t, t-T) \underline{I} .
$$

Thus, we can conclude that the evolution of the gradient of deformation and its derivate tensors evolve as a power law.

If we consider the UCM integral model, Eq. (72), the integral term combines a decreasing exponential function (which approaches zero when $t^{\prime} \rightarrow-\infty$ ) and a power law variation for the Finger's tensor. Thus, we can find a time $t_{i}$ such as:

$$
\int_{-\infty}^{t_{i}} \mathrm{e}^{\left(t^{\prime}-t\right) / \lambda} \underline{B}\left(t, t^{\prime}\right) \mathrm{d} t^{\prime} \leq \epsilon,
$$

with $\epsilon$ a parameter small enough.

Now, we can proceed to prove the equality $\underline{\underline{\tau}}(t)=\underline{\underline{\tau}}(t+T)$ :

$$
\begin{aligned}
\underline{\underline{\tau}}(t+T) & =\frac{\mu}{\lambda} \underline{\underline{I}}+\frac{\mu}{\lambda^{2}} \int_{-\infty}^{t+T} \mathrm{e}^{\left(t^{\prime \prime}-(t+T)\right) / \lambda} \underline{\underline{B}}\left(t+T, t^{\prime \prime}\right) \\
\mathrm{d} t^{\prime \prime} & =\frac{\mu}{\lambda} \underline{\underline{I}}+\frac{\mu}{\lambda^{2}} \int_{t^{*}+T}^{t+T} \mathrm{e}^{\left(t^{\prime \prime}-(t+T)\right) / \lambda} \underline{\underline{B}}\left(t+T, t^{\prime \prime}\right) \mathrm{d} t^{\prime \prime}+\int_{-\infty}^{t+T} \mathrm{e}^{\left(t^{\prime \prime}-(t+T)\right) / \lambda} \underline{\underline{B}}\left(t+T, t^{\prime \prime}\right) \mathrm{d} t^{\prime \prime} .
\end{aligned}
$$

Using the second intermediate result, we can choose a time $t^{*}$ in order to neglect the second integral without the introduction of a significant error:

$$
\underline{\underline{\tau}}(t+T) \approx \frac{\mu}{\lambda} \underline{\underline{I}}+\frac{\mu}{\lambda^{2}} \int_{t^{*}+T}^{t+T} \mathrm{e}^{\left(t^{\prime \prime}-(t+T)\right) / \lambda} \underline{\underline{B}}\left(t+T, t^{\prime \prime}\right) \mathrm{d} t^{\prime \prime} .
$$


Now, we define $t^{\prime}=t^{\prime \prime}-T$ and use the two intermediate results:

$$
\begin{aligned}
\underline{\underline{\tau}}(t+T) & \approx \frac{\mu}{\lambda} \underline{\underline{I}}+\frac{\mu}{\lambda^{2}} \int_{t^{*}+T}^{t+T} \mathrm{e}^{\left(t^{\prime \prime}-(t+T)\right) / \lambda} \underline{\underline{B}}\left(t+T, t^{\prime \prime}\right) \\
\mathrm{d} t^{\prime \prime} & =\frac{\mu}{\lambda} \underline{\underline{I}}+\frac{\mu}{\lambda^{2}} \int_{t^{*}}^{t} \mathrm{e}^{\left(t^{\prime}-t\right) / \lambda} \underline{\underline{B}}\left(t+T, t^{\prime}+T\right) \mathrm{d} t^{\prime} \approx \underline{\underline{\tau}}(t) .
\end{aligned}
$$

Obviously, the extra-stress steady solution at any point of the recirculating flow domain obtained from the differential form of the UCM model (using the numerical procedure previously described, which uses the periodicity condition [14]) coincides exactly with the one obtained with the integral constitutive equation, where the periodicity is implicitly imposed.

\section{Conclusions}

In this paper we have proved that for linear advection problems, which usually describe the constitutive equations of non-Newtonian fluids, the steady solution can sometimes not be found from the steady state of the evolution problem. We have shown that the existence of a steady regime depends on the initial value considered in the evolution algorithm.

We have established that a steady solution exists for a linear multi-mode viscoelastic model. This solution can be computed from the steady solution of the advection equation which governs the evolution of each partial extra-stress associated to the different modes. In the case of non-linear multi-mode models, we need to compute a steady solution for each non-linear advection problem relates to each mode. In this case, the numerical procedure described in [15] can be applied successfully.

Finally, we have also shown that for some integral constitutive models, as is the case of the UCM integral model, the solution obtained in general steady recirculating flows verifies implicitly the periodicity.

\section{Acknowledgements}

We want to thank the "Société Thermo-Rhéo" branch of “Thermo Haake GMBH, Germany" for providing together with the "Association Nationale pour la Recherche Technologique (ANRT)—France", a research position for one of us (G. Chaidron).

\section{References}

[1] G.L. Hand, A theory of anisotropic fluids, J. Fluid Mech. 13 (1962) 33-46.

[2] E.J. Hinch, L.G. Leal, Constitutive equations in suspension mechanics. Part I, J. Fluid Mech. 71 (1975) $481-495$.

[3] E.J. Hinch, L.G. Leal, Constitutive equations in suspension mechanics. Part II, J. Fluid Mech. 76 (1976) 187-208.

[4] F. Meslin, Propriétés Rhéologiques des Composites Fibres Courtes á l'Etat Fondu, PhD thesis, Ecole Normale Superieure de Cachan, 1997.

[5] G.K. Batchelor, The stress generated in non-dilute suspensions in elongated particles by pure straining motion, J. Fluid Mech. 46 (1971) 813.

[6] S.M. Dinh, R.C. Armstrong, A rheological equation of state for semi-concentrated fiber suspensions, J. Rheol. 28 (1984) 207.

[7] Ch.L. Tucker III, Flow regimes for fiber suspensions in narrow gap, J. Non-Newtonian Fluid Mech. 39 (1991) $239-268$. 
[8] S.G. Advani, Ch.L. Tucker III, Closure approximations for three-dimensional structure tensors, J. Rheol. 34 (1990) $367-386$.

[9] R. Keunings, Viscoelastic Flows, in: S.G. Advani (Ed.), Flow and Rheology in Polymer Composites Manufacturing, Elsevier, Amsterdam, 1994.

[10] T.C.B. McLeish, R.G. Larson, Molecular constitutive equations for a class of branched polymers: the Pom-Pom polymer, J. Rheol. 42 (1) (1998) 81-110.

[11] D. Rajagopalan, R.C. Armstrong, R.A. Brown, Calculations of steady viscoelastic flow using a multimode Maxwell model: application of the explicitly elliptic momentum equation formulation, J. Non-Newtonian Fluid Mech. 36 (1990) $135-157$.

[12] F.P.T. Baaijens, Experimental and Numerical Analysis of Viscoelastic Flows, PPS-15, Polymer Processing Society, The Netherlands, 1999.

[13] A. Ammar, F. Chinesta, A. Poitou, 3D Extrusion Simulation of the Pom-Pom Viscoelastic Model, ECCOMAS 2001, Swansea, Wales, UK, 2001.

[14] F. Chinesta, G. Chaidron, On the steady solution of linear advection problems in steady recirculating flows, J. Non-Newtonian Fluid Mech. 98 (2001) 65-80.

[15] G. Chaidron, F. Chinesta, On the Steady Solution of Non-Linear Advection Equations in Steady Recirculating Flows, Computer Methods in Applied Mechanics and Engineering, in press.

[16] K. Chiba, K. Nakamura, Numerical solution of fiber suspension flow through a complex channel, J. Non-Newtonian Fluid Mech. 78 (1998) 167-185.

[17] F. Chinesta, Modélisation Numérique en Mise en Forme de Polyméres et Ceramiques: Differents Problémes de Transport, Habilitation á Diriger des Recherches, Université Paris 6, 2000.

[18] E.A.J.F. Peters, M.A. Hulsen, B.H.A.A. van den Brule, Instationary Eulerian viscoelastic flow using time separable Rivlin-Sawyers constitutive equations, J. Non-Newtonian Fluid Mech. 89 (2000) 209-228. 